\title{
APPROXIMATE HOMOMORPHISMS ON LATTICES
}

\author{
ROMAN BADORA, TOMASZ KOCHANEK, AND BARBARA PRZEBIERACZ
}

\begin{abstract}
We prove two results concerning an Ulam-type stability problem for homomorphisms between lattices. One of them involves estimates by quite general error functions; the other deals with approximate (join) homomorphisms in terms of certain systems of lattice neighborhoods. As a corollary, we obtain a stability result for approximately monotone functions.
\end{abstract}

\section{INTRODUCTION}

More than half a century ago, S.M. Ulam [10] posed the problem of finding conditions which guarantee that any nearly additive map defined, say, on a semigroup must be close to a truly additive map. In 1978, P.M. Gruber [4] reformulated his question by posing a more general stability problem: "Suppose a mathematical object satisfies a certain property approximately. Is it then possible to approximate this object by objects, satisfying the property exactly?'. This initiated a broad research program on the stability problem in theory of functional equations; for more information the reader may consult [5].

In this note, we deal with an Ulam-type problem for homomorphisms of lattices. We present two results where satisfying the homomorphism equation 'approximately' is formalized either with the aid of error functions or in terms of abstractly understood neighborhoods in lattices. In order to justify our approach let us mention a few known results concerning the stability problem in lattices.

A pioneering paper in this context is the one by N.J. Kalton and J.W. Roberts [6] which contains the following deep result (originally formulated for algebras of sets and nearly additive set functions).

Theorem 1 (Kalton \& Roberts [6]). Let $X$ be a Boolean algebra and $f: X \rightarrow \mathbb{R}$ a function satisfying

$$
|f(x \vee y)-f(x)-f(y)| \leqslant 1 \quad \text { for } x, y \in X \text { with } x \wedge y=0 .
$$

Then there exists a map $g: X \rightarrow \mathbb{R}$ such that

$$
g(x \vee y)=g(x)+g(y) \quad \text { for } x, y \in X \text { with } x \wedge y=0
$$

and $|f(x)-g(x)|<45$ for every $x \in X$.

This result is of fundamental importance in functional analysis, especially in theory of twisted sums of quasi-Banach spaces (see, e.g. [7]), as well as in the stability problem for vector meaures (cf. [8]). A somehow related result, very combinatorial in its nature, was obtained by I. Farah.

2010 Mathematics Subject Classification. Primary 06B23, 06D99, 39B82. 
Theorem 2 (Farah [1]). Let $n, m \in \mathbb{N}, X=2^{\{1,2, \ldots, m\}}$ and $Y=2^{\{1,2, \ldots, n\}}$. Suppose that $\varphi: Y \rightarrow[0, \infty]$ is a submeasure, that is, $\varphi(0)=0, \varphi(A) \leqslant \varphi(A \cup B)$, for $A, B \in Y$ and $\varphi(A \cup B) \leqslant \varphi(A)+\varphi(B)$, for $A, B \in Y$. Moreover, we assume that $\phi$ is nonpathological, that is, it is equal to the supremum of all measures it dominates. Let $\varepsilon>0$ and $f: X \rightarrow Y$ be such that

$$
\begin{gathered}
\varphi(f(x \cup y) \div(f(x) \cup f(y))<\varepsilon \quad \text { for } x, y \in X, \\
\varphi(f(X \backslash x) \div(Y \backslash f(x)))<\varepsilon \quad \text { for } x \in X .
\end{gathered}
$$

Then there exists a lattice homomorphism $g: X \rightarrow Y$ such that $\varphi(f(x) \div g(x))<521 \varepsilon$ for every $x \in X$.

In the light of the result above, one seemingly natural approach to the stability problem in lattices would be to assume that a given map $f: X \rightarrow Y$ between lattices $X$ and $Y$ satisfies $f(x \vee y) \div(f(x) \vee f(y)) \leqslant \varepsilon$ for all $x, y \in X$ and some fixed $\varepsilon \in Y$. However, such an approach turns out to be too naive, at least in the case where $Y$ is assumed to be a Boolean algebra. Indeed, just define $g(x)=f(x) \backslash \varepsilon$ and notice that $g$ is then a lattice homorphism satisfying $f(x) \div g(x) \leqslant \varepsilon$ for each $x \in X$. Therefore, we propose two different ways of formalizing the stability problem - one involves error functions instead of the constant factor $\varepsilon$, while the other expresses the closedness of $f(x \vee y)$ to $f(x) \vee f(y)$ in terms of a system of neighborhoods (see Theorems 7 and 8 below, respectively).

Both of our approaches share a common root which is a certain separation, or a "sandwichtype', result (see Lemma 6 below). It is related to some already known separation theorems.

Theorem 3 (Förg-Rob, Nikodem, Páles [2]). Assume that a function $f: \mathbb{R} \rightarrow \mathbb{R}$ is quasiconcave (i.e. $f(x) \geqslant \min \{f(a), f(b)\}$ for all $a \leqslant x \leqslant b$ ), a function $g: \mathbb{R} \rightarrow \mathbb{R}$ is quasiconvex (i.e. $g(x) \leqslant \max \{g(a), g(b)\}$ for all $a \leqslant x \leqslant b$ ) and we have $f(x) \leqslant g(x)$ for every $x \in \mathbb{R}$. Then there exists a monotone map $h: \mathbb{R} \rightarrow \mathbb{R}$ such that $f(x) \leqslant h(x) \leqslant g(x)$ for every $x \in \mathbb{R}$.

W. Kubiś [9] noted that a similar 'sandwich-type' theorem is valid for maps between linearly ordered spaces (see [9, Thm. 2.1]), whereas it fails to hold for maps from $\mathbb{R}^{2}$ to $\mathbb{R}$ and actually even for maps from the four-element Boolean algebra $\{0,1\}^{2}$ to the threeelement linearly ordered space $\{0,1,2\}$. Moreover, Kubiś showed ([9, Thm. 3.3]) that a 'sandwich-type' theorem for the class of $S_{4}$ bi-convexity spaces holds true when the image space is a complete Boolean algebra (being a bi-convexity space with convexities consisting of ideals and filters). The class of $S_{4}$ bi-convexity spaces includes, for example, real vector spaces (for more information consult [11]). As a consequence of this quite abstract version of a separation theorem, Kubiś derived the following corollary (see [9, Thm. 3.7]).

Theorem 4 (Kubiś [9]). Let $L$ be a distributive lattice, $\mathbb{B}$ be a complete Boolean algebra, $f, g: L \rightarrow \mathbb{B}$ and assume that $f$ is a meet homomorphism, $g$ is a join homomorphism and $f(x) \leqslant g(x)$ for $x \in L$. Then there exists a lattice homomorphism $h: L \rightarrow \mathbb{B}$ such that $f(x) \leqslant h(x) \leqslant g(x)$ for every $x \in L$. 
As an application of their Theorem 3, Förg-Rob, Nikodem and Páles showed the following result which yields an Ulam-type stability for monotone maps.

Theorem 5 (Förg-Rob, Nikodem, Páles [2]). Let $I \subset \mathbb{R}$ be an interval, $\varepsilon \geqslant 0$ and assume that a function $f: I \rightarrow \mathbb{R}$ satisfies

$$
\min \{f(x), f(y)\}-\varepsilon \leqslant f(t x+(1-t) y) \leqslant \max \{f(x), f(y)\}+\varepsilon \quad \text { for } x, y \in I, t \in[0,1] \text {. }
$$

Then there exists a monotone function $g: I \rightarrow \mathbb{R}$ such that $|f(x)-g(x)| \leqslant \varepsilon / 2$ for every $x \in I$.

We will see that this theorem can be easily derived from our results on approximate lattice homomorphisms.

\section{Results}

Recall that a lattice is called conditionally complete provided every its bounded subsets admits the least upper bound and the greatest lower bound. A map $f$ between lattices $X$ and $Y$ is called a join homomorphism if it preserves joins, i.e. $f(x \vee y)=f(x) \vee f(y)$ for $x, y \in X$, and it is called a meet homomorphism if it preserves meets, i.e. $f(x \wedge y)=$ $f(x) \wedge f(y)$ for $x, y \in X$. It is called a lattice homomorphism if it is both a join and a meet homomorphism. In the proofs of our stability theorems we shall need the following separation result.

Lemma 6. Let $X$ be a distributive lattice and $Y$ be a conditionally complete lattice. Assume that maps $\Phi, \Psi: X \rightarrow Y$ satisfy the following conditions: $\Phi \leqslant \Psi$,

$$
\begin{aligned}
& \Phi(x \vee y) \leqslant \Phi(x) \vee \Phi(y) \quad \text { for } x, y \in X, \\
& \Psi(x \vee y) \geqslant \Psi(x) \vee \Psi(y) \quad \text { for } x, y \in X .
\end{aligned}
$$

Then there exists a join homomorphism $F: X \rightarrow Y$ such that $\Phi \leqslant F \leqslant \Psi$.

Proof. Notice that for each $x \in X$ and each $z \in X$ with $z \leqslant x$ we have

$$
\Phi(z) \leqslant \Psi(z) \leqslant \Psi(z) \vee \Psi(x) \leqslant \Psi(z \vee x)=\Psi(x) .
$$

Hence, the set $\{\Phi(z): z \leqslant x\}$ is bounded from above and we can define

$$
F(x)=\sup \{\Phi(z): z \leqslant x\} \text { for } x \in X .
$$

Plainly, we have $F(x) \geqslant \Phi(x)$, while (1) implies that also $F(x) \leqslant \Psi(x)$ for every $x \in X$.

Now, fix any $x, y \in X$ and consider an arbitrary $z \leqslant x \vee y$. Since

$$
z=z \wedge(x \vee y)=(z \wedge x) \vee(z \wedge y)
$$

we have

$$
\Phi(z) \leqslant \Phi(z \wedge x) \vee \Phi(z \wedge y) \leqslant F(x) \vee F(y)
$$

From the definition of $F$ we thus get that $F(x \vee y) \leqslant F(x) \vee F(y)$. Moreover, $F$ is a monotone increasing function, therefore, $F(x) \leqslant F(x \vee y)$ and $F(y) \leqslant F(x \vee y)$ which implies that $F(x) \vee F(y) \leqslant F(x \vee y)$ and finishes the proof. 
Notice that by interchanging $\vee$ and $\wedge$ in the lattice $X$ or $Y$ (or both), we can derive three analogous results to Lemma6. Moreover, combining this lemma with the Kubiś result (Theorem 4), we infer that in the case where $L$ is a distributive lattice, $\mathbb{B}$ is a complete Boolean algebra and maps $\Phi_{1}, \Phi_{2}, \Psi_{1}, \Psi_{2}: L \rightarrow \mathbb{B}$ satisfy $\Psi_{2} \leqslant \Phi_{2} \leqslant \Phi_{1} \leqslant \Psi_{1}$ along with the following system of inequalites:

$$
\begin{aligned}
& \Psi_{2}(x \wedge y) \leqslant \Psi_{2}(x) \wedge \Psi_{2}(y) \\
& \Phi_{2}(x \wedge y) \geqslant \Phi_{2}(x) \wedge \Phi_{2}(y) \\
& \Phi_{1}(x \vee y) \leqslant \Phi_{1}(x) \vee \Phi_{1}(y) \\
& \Psi_{1}(x \vee y) \geqslant \Psi_{1}(x) \vee \Psi_{1}(y)
\end{aligned}
$$

for all $x, y \in L$, then there is a lattice homomorphism $H: L \rightarrow \mathbb{B}$ lying between $\Psi_{2}$ and $\Psi_{1}$, i.e. $\Psi_{2} \leqslant H \leqslant \Psi_{1}$.

We are in a position to prove our first stability result.

Theorem 7. Let $X$ and $Y$ be distributive lattices and assume that $Y$ is conditionally complete and satisfies the dual to the infinite distributive law, that is,

$$
y \vee \inf S=\inf \{y \vee s: s \in S\}
$$

for every $y \in Y$ and $S \subset Y$ bounded from below. Assume that maps $f: X \rightarrow Y, \phi, \psi: X \times$ $X \rightarrow Y$ satisfy the following conditions:

$$
\begin{aligned}
& \phi(z, z) \leqslant \phi(x, y) \quad \text { for } x, y, z \in X \text { with } x, y \leqslant z \\
& \psi(x, y) \leqslant \psi(z, z) \quad \text { for } x, y, z \in X \text { with } x, y \leqslant z
\end{aligned}
$$

and

$$
\phi(x, y) \wedge f(x \vee y) \leqslant f(x) \vee f(y) \leqslant f(x \vee y) \vee \psi(x, y) \quad \text { for } x, y \in X .
$$

Then there exists a join homomorphism $F: X \rightarrow Y$ such that

$$
\phi(x, x) \wedge f(x) \leqslant F(x) \leqslant f(x) \vee \psi(x, x) \quad \text { for } x \in X .
$$

Proof. We start by proving that

$$
\phi(x, x) \wedge f(x) \leqslant f\left(x_{1}\right) \vee \ldots \vee f\left(x_{n}\right) \leqslant f(x) \vee \psi(x, x)
$$

for all $x_{1}, \ldots, x_{n} \in X$, where $x=x_{1} \vee \ldots \vee x_{n}$.

We proceed by induction on $n$. For $n=1$ the inequality is obvious. Assume (7) holds for some $n \in \mathbb{N}$ and all $x_{1}, \ldots, x_{n} \in X$. For arbitrary $x_{1}, \ldots, x_{n+1} \in X$ set $x=x_{1} \vee \ldots \vee x_{n}$ and $\bar{x}=x \vee x_{n+1}$. By the induction hypothesis, (5) and (4), we obtain

$$
\begin{aligned}
\left(f\left(x_{1}\right) \vee \ldots \vee f\left(x_{n}\right)\right) \vee f\left(x_{n+1}\right) & \leqslant f(x) \vee \psi(x, x) \vee f\left(x_{n+1}\right) \\
& \leqslant f(\bar{x}) \vee \psi\left(x, x_{n+1}\right) \vee \psi(x, x) \\
& \leqslant f(\bar{x}) \vee \psi(\bar{x}, \bar{x}) .
\end{aligned}
$$


Similarly, using (5) and (3) we get

$$
\begin{aligned}
\left(f\left(x_{1}\right) \vee \ldots \vee f\left(x_{n}\right)\right) \vee f\left(x_{n+1}\right) & \geqslant(f(x) \wedge \phi(x, x)) \vee f\left(x_{n+1}\right) \\
& =\left(\phi(x, x) \vee f\left(x_{n+1}\right)\right) \wedge\left(f(x) \vee f\left(x_{n+1}\right)\right) \\
& \geqslant \phi(x, x) \wedge \phi\left(x, x_{n+1}\right) \wedge f(\bar{x}) \\
& \geqslant \phi(\bar{x}, \bar{x}) \wedge f(\bar{x})
\end{aligned}
$$

which completes the inductive proof of inequality (7).

Define functions $\Phi, \Psi: X \rightarrow Y$ be the formulas

$$
\Phi(x)=\inf \left\{f\left(x_{1}\right) \vee \ldots \vee f\left(x_{n}\right): n \in \mathbb{N}, x_{1}, \ldots, x_{n} \in X, x=x_{1} \vee \ldots \vee x_{n}\right\}
$$

and

$$
\Psi(x)=\sup \left\{f\left(x_{1}\right) \vee \ldots \vee f\left(x_{n}\right): n \in \mathbb{N}, x_{1}, \ldots, x_{n} \in X, x=x_{1} \vee \ldots \vee x_{n}\right\}
$$

Note that these definitions are correct as inequality (7) guarantees that the set under the infimum and the supremum sign is bounded. Note also that the same inequality implies that

$$
\begin{array}{ll}
\phi(x, x) \wedge f(x) \leqslant \Phi(x) \leqslant f(x) & \text { for } x \in X \\
f(x) \leqslant \Psi(x) \leqslant f(x) \vee \psi(x, x) & \text { for } x \in X
\end{array}
$$

Moreover, notice that

$$
\begin{aligned}
& \Phi(x \vee y) \leqslant \Phi(x) \vee \Phi(y) \quad \text { for } x, y \in X ; \\
& \Psi(x \vee y) \geqslant \Psi(x) \vee \Psi(y) \quad \text { for } x, y \in X .
\end{aligned}
$$

Indeed, inequality 10 follows from the assumed dual distributivity law and the fact that for arbitrary $x_{1}, \ldots, x_{n}, y_{1} \ldots y_{m} \in X$ satisfying $x=x_{1} \vee \ldots \vee x_{n}$ and $y=y_{1} \vee \ldots \vee y_{m}$ we have

$$
\Phi(x \vee y) \leqslant\left(f\left(x_{1}\right) \vee \ldots \vee f\left(x_{n}\right)\right) \vee\left(f\left(y_{1}\right) \vee \ldots \vee f\left(y_{m}\right)\right) .
$$

For inequality (11) observe that for any $x_{1}, \ldots, x_{n}$ as above we have

$$
f\left(x_{1}\right) \vee \ldots \vee f\left(x_{n}\right) \leqslant f\left(x_{1}\right) \vee \ldots \vee f\left(x_{n}\right) \vee f(y) \leqslant \Psi(x \vee y)
$$

which yields $\Psi(x) \leqslant \Psi(x \vee y)$. Similarly, we get $\Psi(y) \leqslant \Psi(x \vee y)$, hence inequality (11).

Finally, an appeal to Lemma 6 produces a join homomorphism $F: X \rightarrow Y$ such that $\Phi \leqslant F \leqslant \Psi$. Combining it with (8) and (9) we obtain assertion (6) as desired.

In our next result we express the assumption that $f(x) \vee f(y)$ is 'close' to $f(x \vee y)$ with the aid of a system of lattice neighborhoods.

Theorem 8. Let $X$ and $Y$ be distributive lattices and assume that $Y$ is conditionally complete and satisfies the dual to the infinite distributive law. Assume moreover that there is a function $\mathcal{N}: Y \rightarrow 2^{Y}$, each of whose value is a bounded set, and which satisfies the following conditions:

(i) $y \in \mathcal{N}(y)$ for each $y \in Y$;

(ii) if $t, u \in \mathcal{N}(z)$ and $t \leqslant y \leqslant u$, then $y \in \mathcal{N}(z)$; 
(iii) $\sup \mathcal{N}(y) \in \mathcal{N}(y)$ and $\inf \mathcal{N}(y) \in \mathcal{N}(y)$ for each $y \in Y$;

(iv) if $t \in \mathcal{N}(u)$ and $u \vee y \in \mathcal{N}(z)$, then $t \vee y \in \mathcal{N}(z)$.

Then for every map $f: X \rightarrow Y$ satisfying

$$
f(x) \vee f(y) \in \mathcal{N}(f(x \vee y)) \quad \text { for } x, y \in X
$$

there exists a join homomorphism $F: X \rightarrow Y$ such that $F(x) \in \mathcal{N}(f(x))$ for every $x \in X$.

Proof. By induction we can show that

$$
f\left(x_{1}\right) \vee \ldots \vee f\left(x_{n}\right) \in \mathcal{N}\left(f\left(x_{1} \vee \ldots \vee x_{n}\right)\right)
$$

for all $n \in \mathbb{N}$ and $x_{1}, \ldots, x_{n} \in X$. Indeed, condition (i) gives the assertion for $n=1$. Suppose condition (13) is valid for a fixed $n \in \mathbb{N}$ and let $x_{1}, \ldots, x_{n+1} \in X$. Set

$$
a=f\left(x_{1}\right) \vee \ldots \vee f\left(x_{n}\right), \quad b=f\left(x_{1} \vee \ldots \vee x_{n}\right), \quad c=f\left(x_{n+1}\right), \quad d=f\left(x_{1} \vee \ldots \vee x_{n+1}\right) .
$$

By condition $(12)$, we have $b \vee c \in \mathcal{N}(d)$, whereas our inductive hypothesis gives $a \in \mathcal{N}(b)$. Therefore, by condition (iv) we infer that $a \vee c \in \mathcal{N}(d)$ which completes the inductive proof of 13 .

Now, we define maps $\Phi, \Psi: X \rightarrow Y$ as in the previous proof, that is,

$$
\begin{aligned}
& \Phi(x)=\inf \left\{f\left(x_{1}\right) \vee \ldots \vee f\left(x_{n}\right): n \in \mathbb{N}, x_{1}, \ldots, x_{n} \in X, x=x_{1} \vee \ldots \vee x_{n}\right\}, \\
& \Psi(x)=\sup \left\{f\left(x_{1}\right) \vee \ldots \vee f\left(x_{n}\right): n \in \mathbb{N}, x_{1}, \ldots, x_{n} \in X, x=x_{1} \vee \ldots \vee x_{n}\right\} .
\end{aligned}
$$

Note that, by (13), every element of the set under the infimum/supremum sign belongs to $\mathcal{N}(f(x))$. Hence, assumptions (ii) and (iii) imply that

$$
\Psi(x), \Phi(x) \in \mathcal{N}(f(x)) \text { for } x \in X .
$$

Moreover, we have $\Phi \leqslant f \leqslant \Psi$ and similarly as in the proof of Theorem 7 we show that $\Phi(x \vee y) \leqslant \Phi(x) \vee \Phi(y)$ and $\Psi(x \vee y) \geqslant \Psi(x) \vee \Psi(y)$ for all $x, y \in X$.

In view of Lemma 6, there is a join homomorphism $F: X \rightarrow Y$ such that $\Phi \leqslant F \leqslant \Psi$. Finally, by (14) and assumption (ii), we obtain $F(x) \in \mathcal{N}(f(x))$ for every $x \in X$.

Notice that by interchanging $\vee$ and $\wedge$ in $X$ or $Y$ (or both) we can obtain three analogous results to Theorems 7 and 8 .

Example 9. Condition (ii) above claims, in the lattice terminology, that each $\mathcal{N}(z)$ is convex. By (iii), we require that it is closed under the sup/inf operations. Condition (iv) seems a bit demanding, however, it is satisfied in some natural situations as the examples below show.

(a) Assume $Y$ has the minimal element. For each $z \in Y$ define $\mathcal{N}(z)=\{y \in Y: y \leqslant z\}$ which is nothing else but the ideal generated by $\{z\}$. It is easily verified that the function $Y \ni z \mapsto \mathcal{N}(z)$ satisfies all the axioms (i)-(iv).

(b) For any $n \in \mathbb{N}$, consider the lattice $\mathbb{L}_{n}$ consisting of all natural divisors of $n$, that is, $\left(\mathbb{L}_{n}, \vee, \wedge\right)$ is given as $\mathbb{L}_{n}=\{k \in \mathbb{N}: k \mid n\}$, where $\vee$ is the least common multiple and $\wedge$ is the greatest common divisor. For $z \in \mathbb{L}_{n}$ define $\mathcal{N}(z)$ as the family of those elements of $\mathbb{L}_{n}$ 
whose each prime factor is also a prime factor of $z$. Again, the function $\mathbb{L}_{n} \ni z \mapsto \mathcal{N}(z)$ satifies (i)-(iv).

(c) We can repeat the same idea as above for every finite distributive lattice $Y$. Let $H: Y \rightarrow \mathbb{L}_{n}$ be a one-to-one homomorphism, for a suitable $n \in \mathbb{N}$ (see, e.g., [3, Ch. II.1]), and for every $z \in Y$ define $\mathcal{N}(z)$ as the family of those $y \in Y$ for which we have $H(y) \in$ $\mathcal{N}(H(z))$ in the sense of the definition from the previous example.

(d) Let $\Theta \subset Y^{2}$ be a congruence relation (see [3, Ch. I.3]) and assume it is conditionally complete in the sense that if $y_{i} \equiv z_{i}(\Theta)$ for $i \in I$, then both $\left\{y_{i}: i \in I\right\}$ and $\left\{z_{i}: i \in I\right\}$ are bounded, and we have $\bigvee_{i \in I} y_{i} \equiv \bigvee_{i \in I} z_{i}(\Theta)$ and $\bigwedge_{i \in I} y_{i} \equiv \bigwedge_{i \in I} z_{i}(\Theta)$. For $z \in Y$ define $\mathcal{N}(z)$ to be the abstraction class determined by $z, \mathcal{N}(z)=\{y \in Y: y \equiv z(\Theta)\}$. Conditions (i)-(iv) are then satisfied. Indeed, (i) is trivial; (ii) is valid since every abstraction class forms a convex sublattice (see [3, Lemma I.3.7]); (iii) follows from the conditional completeness of $\Theta$; (iv) follows from the fact that $\Theta$, as every congruence, preserves the join operation.

\section{Approximate monotonicity}

In this section we will show how our theorem can be applied to obtain a stability result for approximately monotone functions. First, note that obviously for any $D \subset \mathbb{R}$ a function $f: D \rightarrow \mathbb{R}$ is increasing if and only if $\max \{f(x), f(y)\}=f(\max \{x, y\})$ for all $x, y \in D$.

Corollary 10. Let $D \subset \mathbb{R}, \varepsilon \geqslant 0$ and assume that a function $f: D \rightarrow \mathbb{R}$ satisfies

$$
\max \{f(x), f(y)\}-f(\max \{x, y\}) \leqslant \varepsilon \quad \text { for } x, y \in D \text {. }
$$

Then there exists an increasing function $g: D \rightarrow \mathbb{R}$ such that $|f(x)-g(x)| \leqslant \varepsilon / 2$ for every $x \in D$.

Proof. We consider the lattices $X=D$ and $Y=[-\infty, \infty]$ in which the join and the meet operations are defined by $x \vee y=\max \{x, y\}$ and $x \wedge y=\min \{x, y\}$. Obviously, $X$ and $Y$ are distributive, $Y$ is also conditionally complete and satisfies the dual to the infinite distributive law.

Define $\phi, \psi: X \times X \rightarrow Y$ by

$$
\phi(x, y)=-\infty \quad \text { and } \quad \psi(x, y)=\sup \{f(z): z \leqslant x\} \vee \sup \{f(z): z \leqslant y\} .
$$

Inequality (3) is then obvious. Note that for all $x, y, z \in X$ with $x \leqslant z$ and $y \leqslant z$ we have

$$
\psi(x, y) \leqslant \sup \{f(u): u \leqslant z\}=\psi(z, z)
$$

which means that inequality (4) is also satisfied.

Now, we shall verify that inequalities (5) are satisfied. The left one is obvious. For the right one, fix any $x, y \in X$ and assume with no loss of generality that $y \leqslant x$. Then

$$
f(x) \leqslant f(x) \vee \psi(x, y)=f(x \vee y) \vee \psi(x, y)
$$

and

$$
f(y) \leqslant \sup \{f(z): z \leqslant x\} \leqslant \psi(x, y) \leqslant f(x \vee y) \vee \psi(x, y)
$$

as desired. 
By Theorem 7, there is a join homomorphism (i.e. an increasing function) $F: X \rightarrow Y$ which satisfies condition (6). Recall that the map $F$ was defined by the formula $F(x)=$ $\sup \{\Phi(z): z \leqslant x\}$, where

$$
\Phi(x)=\inf \left\{f\left(x_{1}\right) \vee \ldots \vee f\left(x_{n}\right): n \in \mathbb{N}, x_{1}, \ldots, x_{n} \in X, x=x_{1} \vee \ldots \vee x_{n}\right\}
$$

(cf. the proofs of Lemma 6 and Theorem 7). From (6) and (16) we get

$$
F(x) \leqslant f(x) \vee \psi(x, x)=f(x) \vee \sup \{f(z): z \leqslant x\} \leqslant f(x)+\varepsilon \quad \text { for } x \in X .
$$

Notice that if $x=x_{1} \vee \ldots \vee x_{n}$ for some $x_{1}, \ldots, x_{n} \in X$, then $x=x_{i}$ for some $i \in\{1, \ldots, n\}$. Hence, by the very definition, we have $\Phi(x) \geqslant f(x)$ and therefore $F(x) \geqslant \Phi(x) \geqslant f(x)$ for each $x \in X$. We have shown that $F$ is an increasing, real-valued function such that $f(x) \leqslant$ $F(x) \leqslant f(x)+\varepsilon$ for each $x \in X$. It remains to define $g: X \rightarrow Y$ as $g(x)=F(x)-\varepsilon / 2$.

Observe that interchanging $\vee$ and $\wedge$ in the above proof we obtain an analogous result on approximately decreasing functions.

Corollary 11. Let $D \subset \mathbb{R}, \varepsilon \geqslant 0$ and assume that a function $f: D \rightarrow \mathbb{R}$ satisfies

$$
f(\max \{x, y\})-\min \{f(x), f(y)\} \leqslant \varepsilon \quad \text { for } x, y \in D .
$$

Then there exists a decreasing function $g: D \rightarrow \mathbb{R}$ such that $|f(x)-g(x)| \leqslant \varepsilon / 2$ for every $x \in D$.

Finally, notice that Theorem 5 can be easily derived from Corollaries 10 and 11, as well as these two corollaries can be derived from Theorem 5 (in the case where $D$ is an interval).

Acknowledgement. The research of the third-named author is a part of the Iterative functional equations and real analysis program (Institute of Mathematics, University of Silesia, Katowice, Poland).

\section{REFERENCES}

[1] I. Farah, Approximate homomorphisms, Combinatorica 18 (1998), 335-348.

[2] W. Förg-Rob, K. Nikodem, Zs. Páles, Separation by monotonic functions, Math. Pannon. 7 (1996), 191-196.

[3] G. Grätzer, General Lattice Theory, Academic Press, New York-San Francisco 1978.

[4] P.M. Gruber, Stability of isometries, Trans. Amer. Math. Soc. 245 (1978), 263-277.

[5] D.H. Hyers, G. Isac, Th.M. Rassias, Stability of functional equations in several variables, Birkhäuser, Basel 1998.

[6] N.J. Kalton, J.W. Roberts, Uniformly exhaustive submeasures and nearly additive set functions, Trans. Amer. Math. Soc. 278 (1983), 803-816.

[7] N. Kalton, Quasi-Banach spaces, in: Handbook of the geometry of Banach spaces, Vol. 2, 1099-1130, North-Holland, Amsterdam 2003 (editors W.B. Johnson and J. Lindenstrauss).

[8] T. Kochanek, Stability of vector measures and twisted sums of Banach spaces, J. Funct. Anal. 264 (2013), 2416-2456.

[9] W. Kubiś, A sandwich theorem for convexity preserving maps, Tatra Mt. Math. Publ. 24 (2002), 125131.

[10] S.M. Ulam, A collection of mathematical problems Wiley-Interscience (Problems in modern mathematics, Science Edition), Interscience, New York, 1960 (1964).

[11] M. van de Vel, Theory of convex structures, North-Holland, Amsterdam 1993. 
Institute of Mathematics, University of Silesia, Bankowa 14, 40-007 Katowice, Poland E-mail address: robadora@ux2.math.us.edu.pl

Institute of Mathematics, Polish Academy of Sciences, Śniadeckich 8, 00-656 Warsaw, Poland and Institute of Mathematics, University of Warsaw, Banacha 2, 02-097 Warsaw, POLAND

E-mail address: tkoch@impan.pl

Institute of Mathematics, University of Silesia, Bankowa 14, 40-007 Katowice, Poland E-mail address: barara.przebieracz@us.edu.pl 